\title{
Discrete Material Flow MANAgement in Multipolar MANUFACTURING NETWORKS
}

\author{
POPA, C. L. \& COTET, C. E.
}

Abstract: In the paper we will present a multipolar manufacturing network simulation using a new approach in discrete material flow management. We validate this aproach using a case study of three partners network acting in a virtual enterprise architecture, analyzed in order to optimize the manufacturing design. Two of them represent product suppliers, and the third represents the enterprise where the assembling will take place, after which the final product will result. In order to evaluate the architecture of the system, we used simulation models of the material flow for the three FMS, using the Witness software. The purpose of the simulation is to introduce our new methodology for multipolar distributed simulation of the material flow in the manufacturing networks in order to optimise the activity between partners and to increase the profit.

Key words: manufacturing networks, multipolar simulation, Witness, FMS
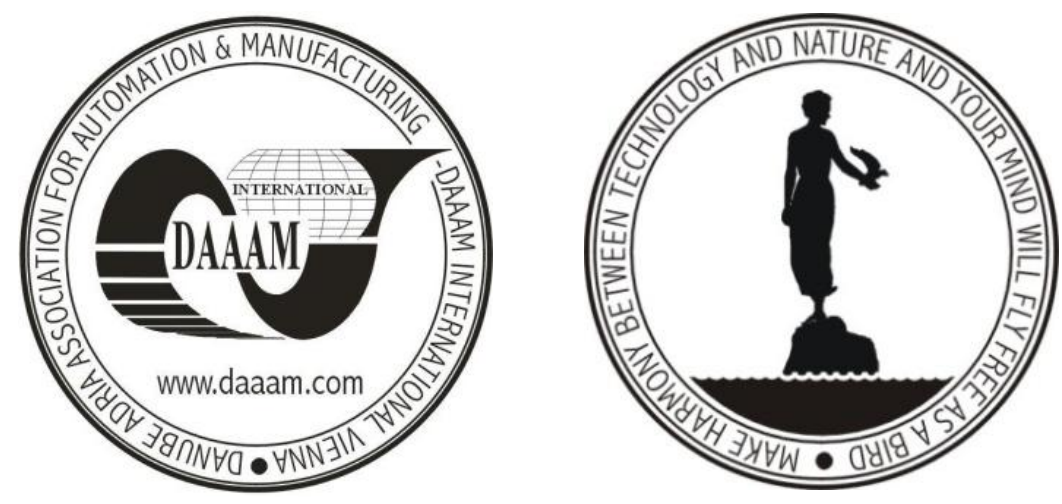

Authors' data: Lecturer Dr. Eng. Popa, C[icerone] L[aurentiu]; Associate Professor Dr. Eng. Cotet, C[ostel] E[mil], University Politehnica of Bucharest, IMST Faculty, MSP Department, UPB-PREMINV Research Centre, 313, Splaiul Independentei, Sector 6, 060042, Bucharest, Romania, laur.popa79@gmail.com, costel@mix.mmi.pub.ro

This Publication has to be referred as: Popa, C[icerone] L[aurentiu] \& Cotet, C[ostel] E[mil] (2010). Discrete Material Flow Management in Multipolar Manufacturing Networks, Chapter 59 in DAAAM International Scientific Book 2010, pp. 679-686, B. Katalinic (Ed.), Published by DAAAM International, ISBN 978-3901509-74-2, ISSN 1726-9687, Vienna, Austria

DOI: $10.2507 /$ daaam.scibook.2010.59 


\section{Introduction}

Our new discrete material flow algorithm is meant to be used in virtual enterprises architectures. The virtual enterprise represents a temporary alliance of enterprises who wish to share resources and aptitudes for the purpose of making a product in the shortest time possible, at the smallest price possible, and with the maximum satisfaction of the client (Camarinha-Matos et al., 1997). It is based on a technical infrastructure, represented by the informational technologies and by the computer communication networks (Dragoi, 2005).

Also we can say that virtual enterprises could be defined as ephemeral organizations in which several companies collaborate to produce a single product during a project cycle time (Tichkiewitch \& al., 2006).

Thus, the projection, planning of production and marketing, supplying, fabrication, services etc. can be realized anywhere in the country, the continent or the world, because of the facilities offered by the infrastructures which allow the change of information, goods and services.

\section{Multipolar distributed simulation}

The multipolar distributed simulation can be defined as an integrated system of monitoring more than two material flow simulations, interconnected inside the architecture of a virtual enterprise (Popa \& Cotet, 2008).

In order to realize a simulation model of the material flow in the system, the first step is to define the necessary machine-tools, the work-pieces and the parts involved in the process on the level of each FMS (Flexible Manufacturing Systems). The inputs in the system are represented by the parameters of the machine-tools, of the work-pieces and of the parts involved, and the output of the system is represented by the optimized architecture of the system. The main limitations of the system are: the realization plans, the available realization stages, and the capacity of the CADCAM-CAE system to analyze the behaviour of the machine-tools, the work-pieces and the parts in the work regime (Lee, 1999).

The purpose of using this method is to achieve an optimum configuration for the system, regarding machines placement in the working place, the parts manufacturing order etc. In the following case study three partners are found, organized under the shape of a virtual organization. Two of them represent product suppliers, and the third represents the enterprise where the assembling will take place, after which the final product will result.

Using specific solutions, each partner can realize a simulation in order to check the capability of its own system to fulfil the function traced in the virtual enterprise. After this, all these simulations can be integrated in a model of multipolar simulation in order to optimize the material flow in the entire system.

In the following we will define the three partners implicated in the system as being the three flexible fabrication systems, and thus FMS1 and FMS2 will represent the components supplier partners and FMS3 will be the system that makes their assembly. 


\section{Case study}

In order to evaluate the architecture of the system, we used simulation models of the material flow for the three FMS, using the Witness software. The simulation of this project was realized in order to demonstrate and confirm the productivity of a fabrication process, based on the presentation of the proposed design and the operational data. Another purpose of the simulation was the identification of the ways to improve the configuration of the system in order to raise productivity.

The improving of productivity means the identification of the material flow concentrators for each FMS and for the manufacturing network as a system, and the proposal of another manufacturing architecture that eliminates the concentrators.

According to the previous statements, FMS1 and FMS2 are diffused systems, and FMS3 is a concentrated system (Cotet \& Dragoi, 2003). Therefore, a concentrated FMS can be defined as a manufacturing architecture based on a single work point surrounded \& assisted by transport, transfer \& deposit facilities. A diffused FMS system can be defined as a manufacturing architecture with more than two work points connected by transport \& transfer systems and using deposits at local or system level.

First we defined the parameters for each machine and conveyor and, after running the simulation we will determine the place of the concentrators. Thus for the first system according to reports we have indentified the blockages at machine M1_2; the activity for this machine is blocked $50 \%$ of the time, $47 \%$ of the time is working, and $3 \%$ of the time is waiting for parts.

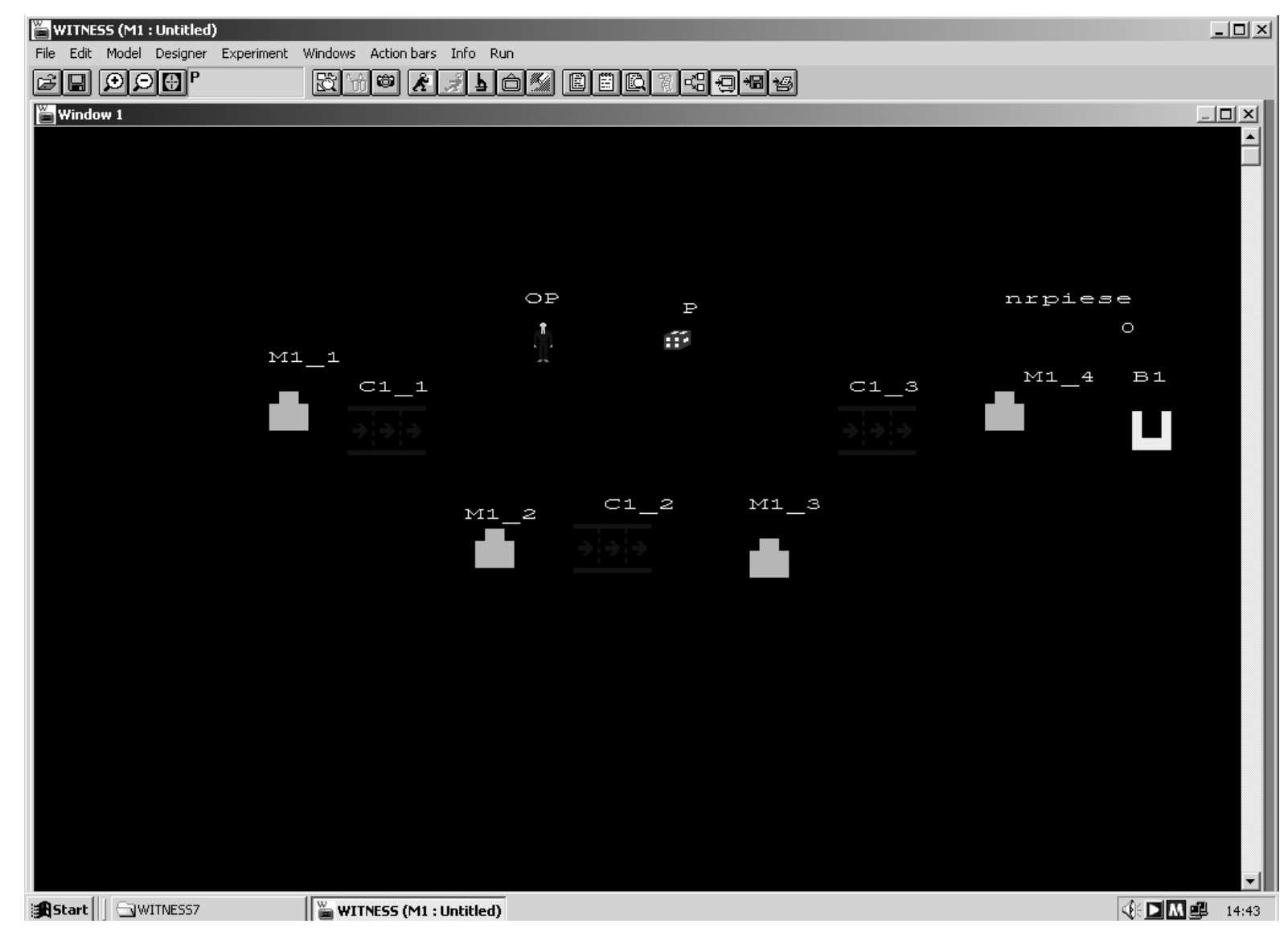

Fig. 1. Witness modelling for FMS1 
The activity reports show blockages in C1_2, its activity is blocked for $45 \%$ of the time, $45 \%$ of the time is working, and $10 \%$ of the time is waiting for parts. Due to the blockages from the other machines M1_4 is working just $11 \%$ of the total time, $89 \%$ of the time is waiting for parts.

Thus, the system appears to be inefficient. We must do a remodelling of the system, a new parameterisation of the system's components and eventually we can run a new simulation. After running the simulation the material flow concentrator from M1_2 machine is eliminated.

In this new configuration M1_2 machine is working $75 \%$ of the total time and $25 \%$ of the time is waiting for parts. An important improvement can be noticed for M1_4 machine, the machine is functioning $98 \%$ of the time, a big difference from the first case when it functioned only $11 \%$ of the time.

In figure 2 the FMS2 is presented during simulation. The parameters for each machine and conveyor were defined, and following the simulation, the place where the concentrators of the respective systems were identified.

The report regarding the activity of conveyor $\mathrm{C} 2 \_1$ is found in figure 3 . We can observe the blocking of activity at this unit for $45 \%$ of the time, the conveyor is stopped for the intervention of the operator $5 \%$ of the time, and the machine works normally just $50 \%$ of the total time.

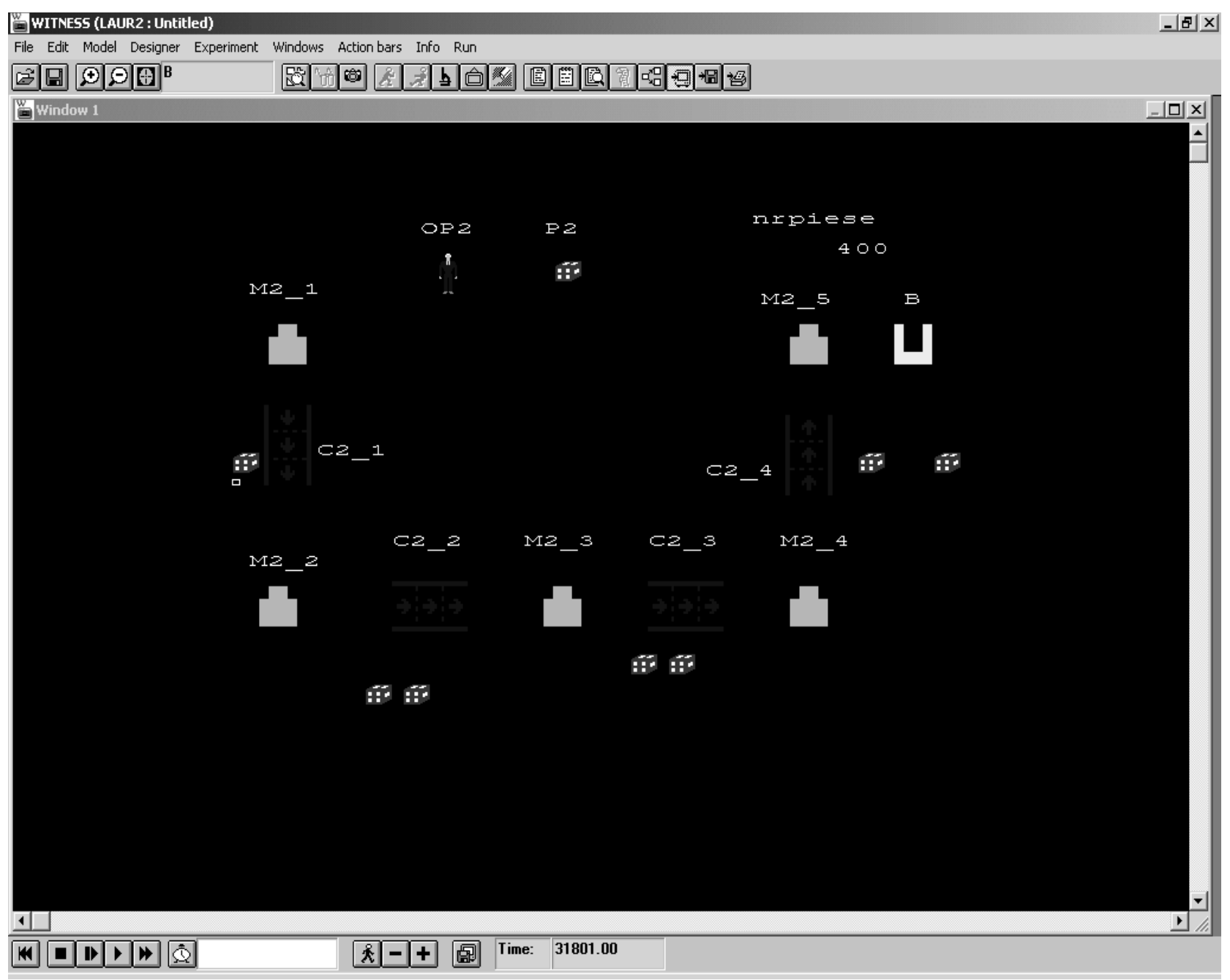

Fig. 2. The FMS2 during simulation 


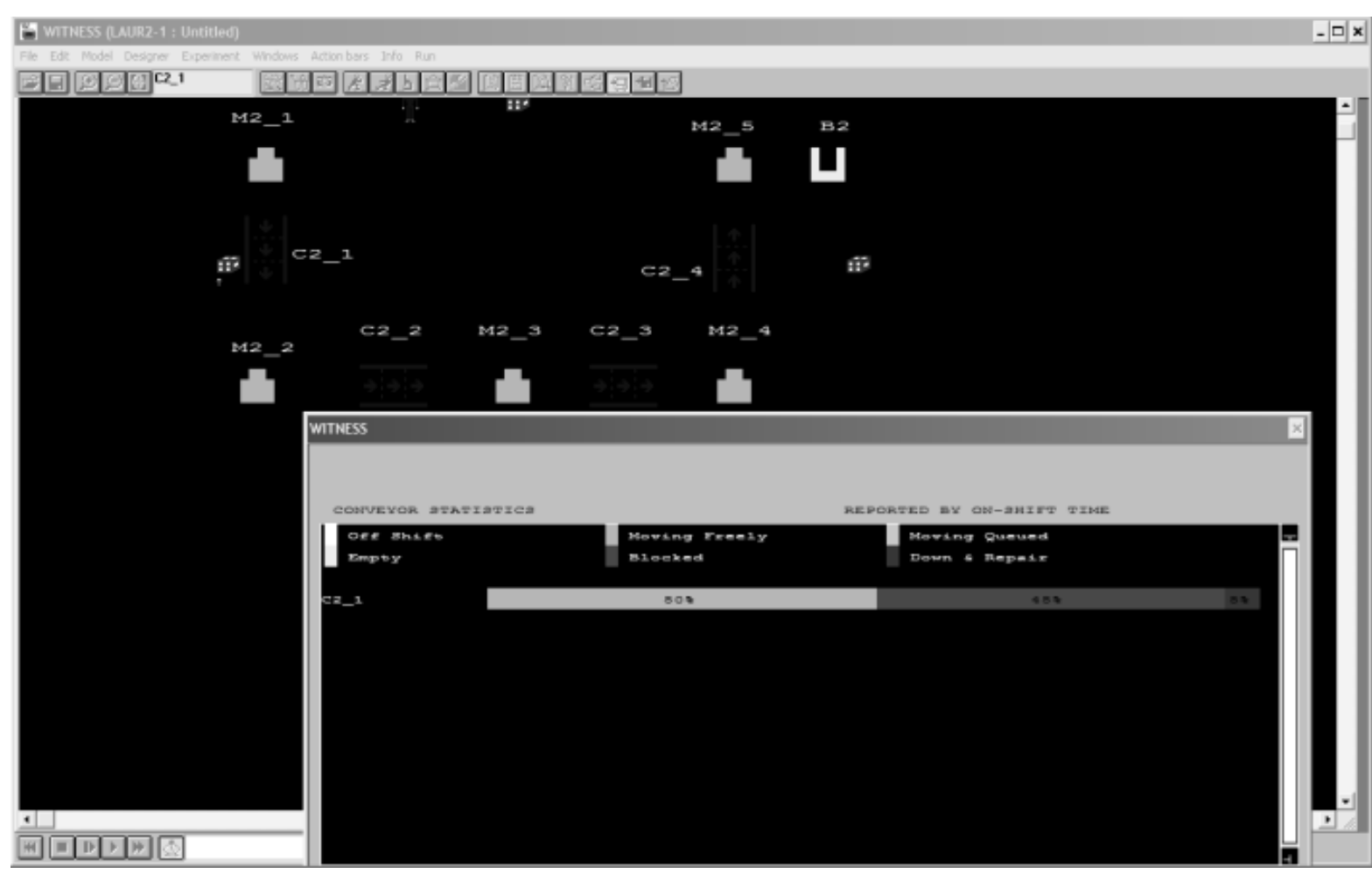

Fig. 3. The report regarding the activity of conveyor C2_1

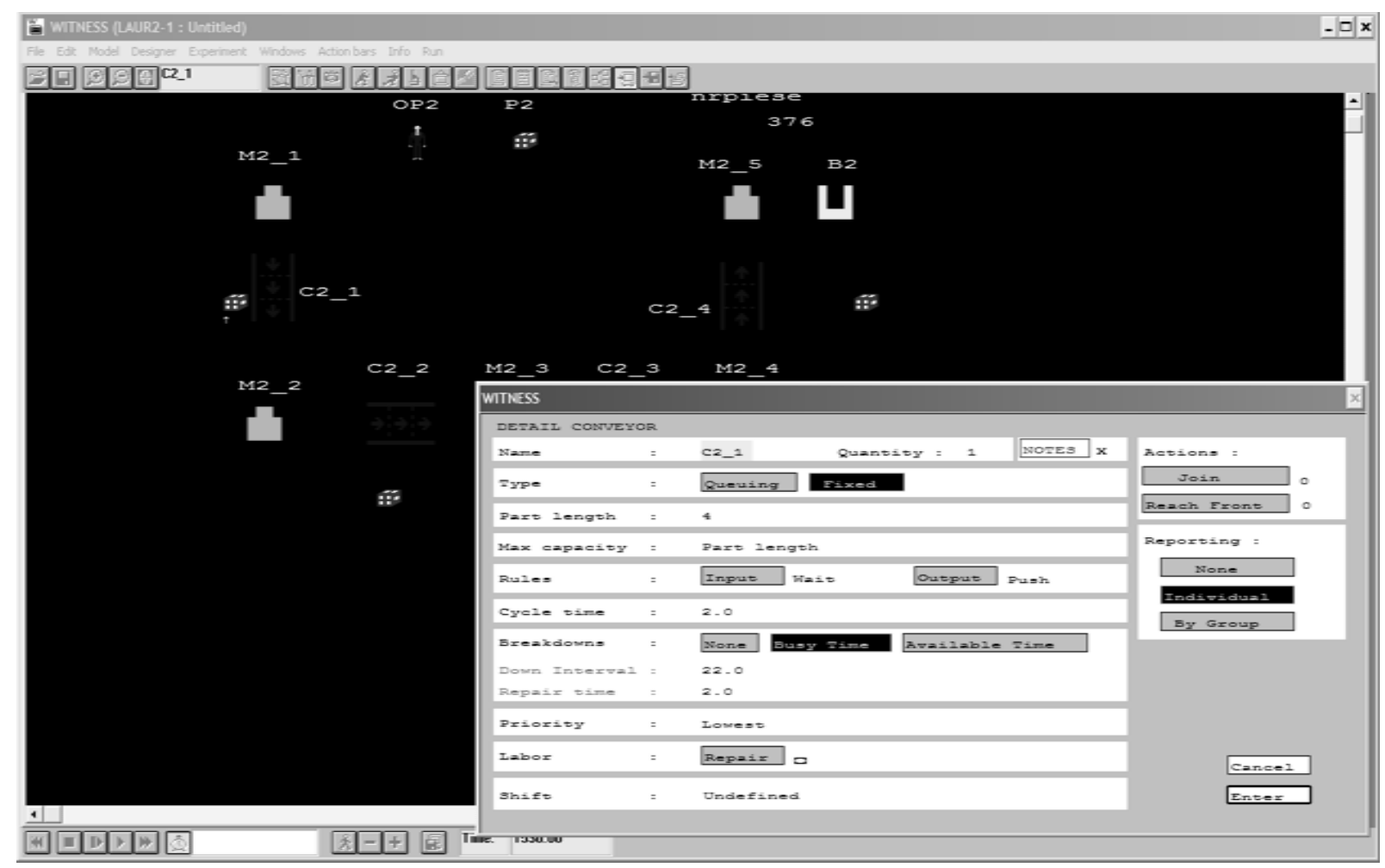

Fig. 4. Definition of parameters for conveyor C2_1

The FMS2 system was remodelled and a new simulation of the material flow in the system was made. In figure 5 the report regarding the activity of conveyor C2_1 after the remodelling is presented and we can observe the total disappearance of blockages found in the previous simulation. Thus, the conveyor is in function $55 \%$ of the time, $34 \%$ of the time it waits for parts arrival, and the time necessary for the intervention of the human resource is of $11 \%$. 
Popa, C.L. \& Cotet, C.E.: Discrete Material Flow Management in Multipolar ...

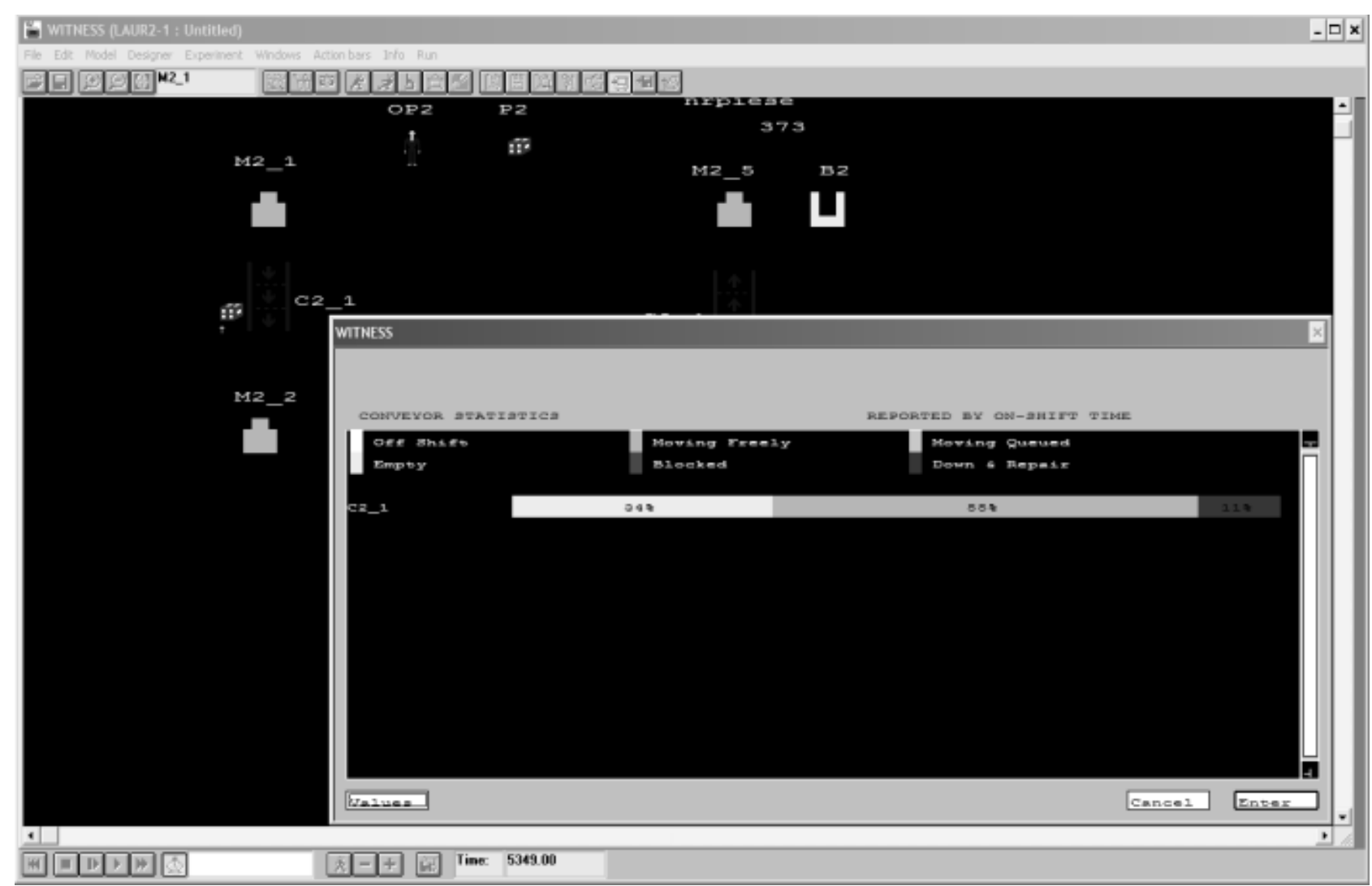

Fig. 5. The report regarding the activity of conveyor C2_1 after system remodelling

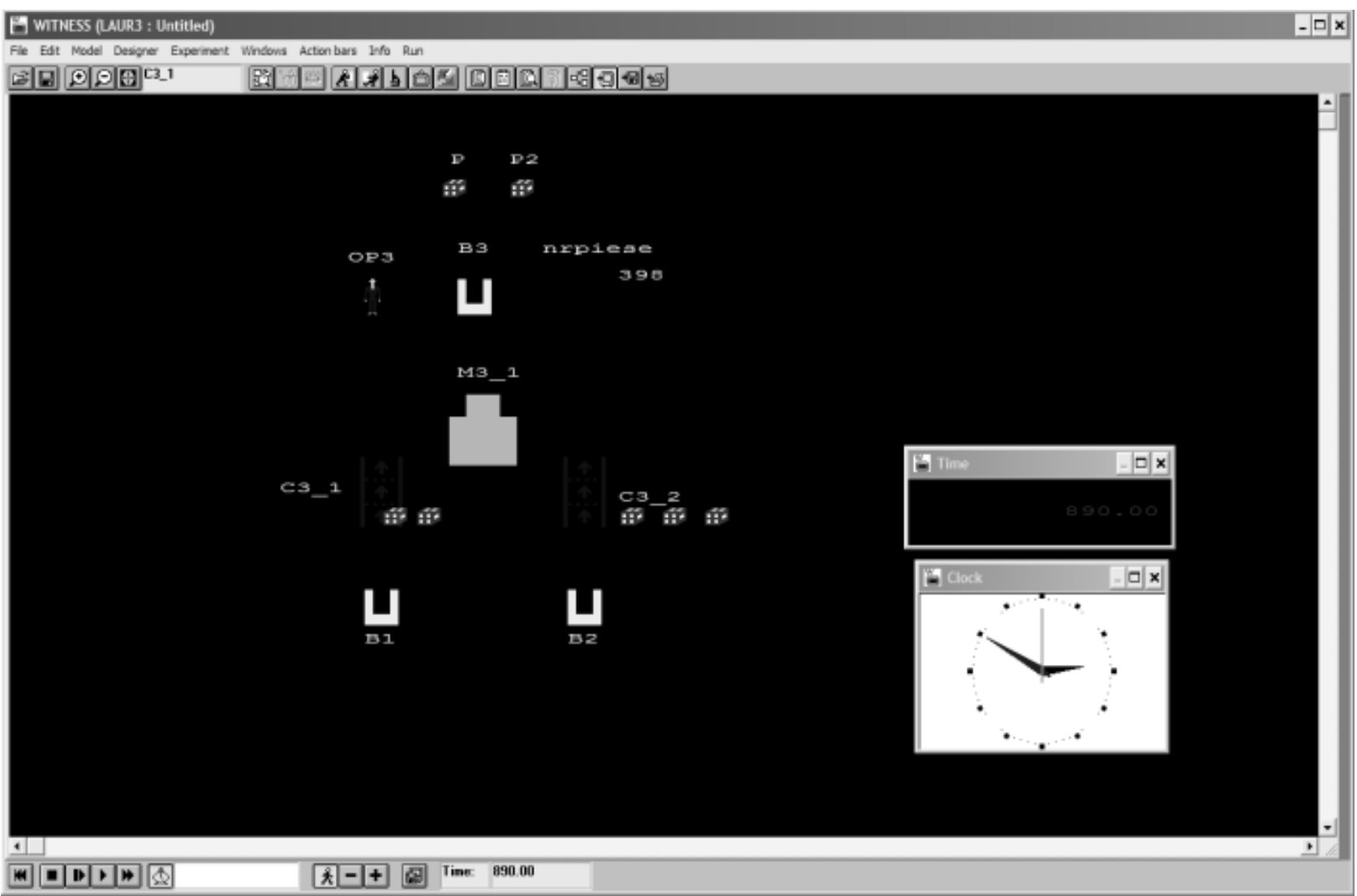

Fig. 6. The FMS3 during simulation

In figure 6 the third manufacturing system is presented during the simulation. We can observe: the B1 and B2 buffers that are the inputs in system 3 and also the 
outputs of system 1 and 2; the two conveyors and the machine on which the final assembly is done (M3_1).

Also, the two parts (P and P2) can be found, which come from the previous systems FMS1 and FMS2, the operator and the number of pieces being worked at the time of reporting. From the study of the rapports and from the localization of the concentrators, especially in conveyor C3_2, results the necessity of remodelling the system for eliminating the concentrators.

This will be done for system 3, and if concentrators will be maintained it results the necessity of intervention over the inputs in the system, and therefore over systems 1 and 2, which through the results of their own outputs determine the two inputs of system 3.

In the case of conveyor C3_2, according to the report presented in figure 7, the existing blockage is reduced from $84 \%$ to $19 \%$ of time. Thus, it results that it works normally $80 \%$ of the time, which indicates a major improvement of its activity and implicitly of that of the system.

After analysing the remodelled system 3 we noticed an increasing of productivity for system 3 and for the whole manufacturing network obtained by optimising the material flow from the three systems separately, but as a general system.

By eliminating the concentrator from conveyor C3_2 we decreased the transfer and transport times for parts on M3_1 machine. The two components were delivered faster so the machine was reprogrammed to assembly faster. So the manufacturing cycle was significantly reduced.

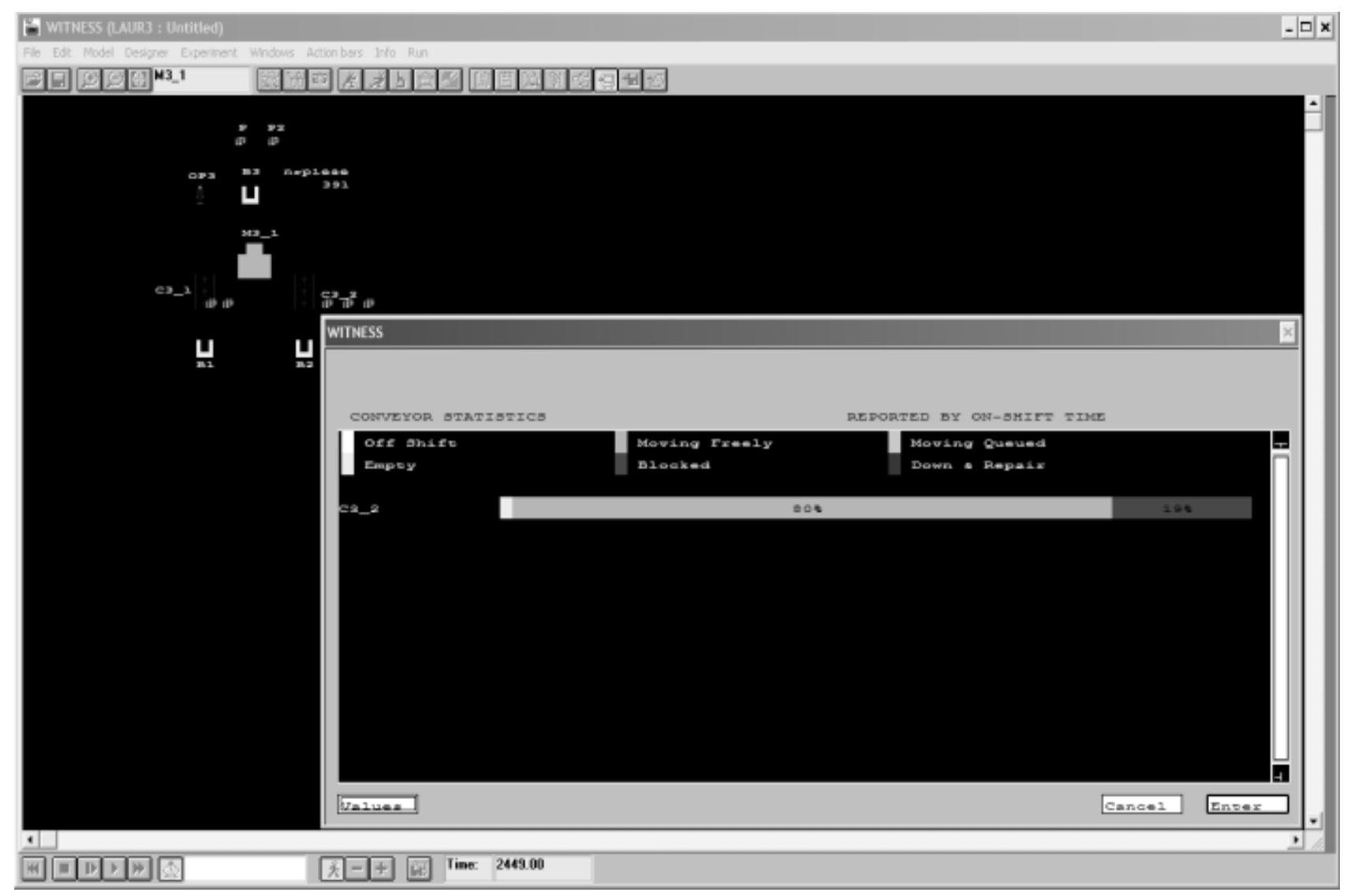

Fig. 7. The report regarding the activity of conveyor C3_2 after system remodelling 


\section{Conclusion}

If we want to optimize the material flow inside a manufacturing network, a simple simulation is not enough. This is the reason why we proposed as a solution, the multipolar simulation, capable of evaluating the performances on a manufacturing network as an integrated system. The analysis of the multipolar simulation does much more than just concatenates the results of isolated simulations. The flow concentrator which results from the multipolar simulation can be any of the concentrators of isolated simulations, but it can also be a totally different one. Some of the main causes of this particularity are the difference in algorithms for diffuse, isolated algorithms. For our case study, eliminating the material flow concentrator of FMS3 improves the production at the level of the entire virtual enterprise, but the most influent concentrator of the entire system is localized in FMS2.

When calculating the necessary transportation time between the two supplier units and the enterprise where the assembly takes place because the working points are geographically de-located, the distances can be extremely large and it's hard to estimate a medium transportation time. Medium times were used for our case study, because the results are relevant, opposed to cases of parts transportation between systems were medium times can't be used because it varies from hours to weeks, depending on problems occurred on roads and distances. However, in our future research we intent to calculate the transportation times using specific distribution laws in order to develop our algorithm and cover more complex case studies.

\section{References}

Camarinha-Matos, L.M.; Carelli, R.; Pellicer, J.; Martín, M. (1997). Towards the virtual enterprise in food industry, Proceedings of the ISIP'97 OE/IFIP/IEEE International. Conference on Integrated and Sustainable Industrial Production, ISBN 0-412-79950-2, Portugal, May 1997, Chapman \& Hall, Lisboa

Cotet, C.E.; Dragoi, G. (2003). Material flow management in validating concentrate and diffused FMS architectures. International Journal of Simulation Modelling IJSIMM, Vol. 2, No. 4, December 2003, pp.109-120, ISSN 1726-4529

Dragoi, G. (2005). Informational Infrastructure of modern enterprise (Infrastructura informatională si de comunicatii a intreprinderii moderne), Ed. Politehnica Press, ISBN 973-8449-73-1, Bucharest

Lee, K. (1999). Principles of CAD/CAM/CAE Systems, Addison Wesley Longman, Inc., ISBN 0-201-38036-6, USA

Popa, C.L.; Cotet C.E. (2008). Multipolar distributed material flow simulation of a virtual enterprise, Proceedings of The 19th International DAAAM SYMPOSIUM, „Intelligent Manufacturing \& Automation:Focus on Next Generation of Intelligent Systems and Solutions, Katalinic, B. (Ed.), pp. 11031104, ISBN 978-3-901509-68-1, ISSN 1726-9679, Published by DAAAM International, Vienna

Tichkiewitch, S.; Radulescu, B. \& Dragoi, G. (2006). Knowledge management for a cooperative design system, Advances in Design, ElMaraghy \& Hoda A. Eds., pp. 97-110, Springer Verlag, ISBN 1-84628-004-4 